\title{
Etnografía del pensamiento del profesorado de matemática de Santiago de Cuba
}

Ethnography of the thought of mathematic teachers in Santiago de Cuba

\author{
Volumen 19, Número 3 \\ Setiembre-Diciembre \\ pp. 1-29
}

\author{
Osmany Justis Katt \\ Camelia Virgen Galán Segura
}

Citar este documento según modelo APA

Justis Katt, Osmany. y Galán Segura, Camelia Virgen. (2019). Etnografía del pensamiento del profesorado de matemática de Santiago de Cuba. Revista Actualidades Investigativas en Educación, 19(3), 1-29. Doi. 10.15517/aie.v19i3.38813 


\title{
Etnografía del pensamiento del profesorado de matemática de Santiago de Cuba \\ Ethnography of the thought of mathematic teachers in Santiago de Cuba
}

\author{
Osmany Justis Katt ${ }^{1}$ \\ Camelia Virgen Galán Segura²
}

\begin{abstract}
Resumen: Este artículo señaló como problema las insuficiencias en la calidad del aprendizaje del alumnado en matemática manifestadas en los bajos resultados de los exámenes de ingreso a la Educación Superior en la provincia de Santiago de Cuba. La indagación no encontró antecedentes de estudios etnográficos en el contexto educativo santiaguero, en consecuencia, se plantea como objetivo realizar un acercamiento etnográfico a la forma en que el profesorado de matemática concibe el diseño de su gestión pedagógica para generar conocimientos duraderos en el alumnado. Se utilizó metodología de tipo cualitativa y el método fundamental para la obtención de información fue el trabajo de campo en una población de 229 personas docentes, de las que fueron muestra 83. Esta investigación se desarrolló desde septiembre 2016 hasta octubre 2017. El empleo de técnicas etnográficas complementó la metodología para lograr como resultado principal una matriz cultural-pedagógica-operatoria para la acción educativa en la enseñanza de la matemática. Asimismo reveló limitaciones teóricas en la comprensión del alcance de la gestión pedagógica del profesorado en el modelo actuante en Santiago de Cuba. La matriz cultural-pedagógica-operatoria es un resultado que creó condiciones metodológicas para la transferencia de buenas prácticas en el aprendizaje de la matemática y abre un espacio de reflexión en la manera de gestionar el aprendizaje.
\end{abstract}

Palabras clave: docente, enseñanza de las matemáticas, pensamiento, etnografía.

\begin{abstract}
This paper focuses as a problem inadequacies in the quality of student learning in mathematics revealed in the low results of the entrance exams to Higher Education in the province of Santiago de Cuba. The research found no antecedent ethnographic studies in the educational context of Santiago, as a consequence, it is proposed as an objective: to develop an ethnographic approach in regard to the way in which the teachers of mathematics conceive the design of their pedagogical management to produce lasting knowledge in the students. The methodology used supports a qualitative approach, constituting an ethnographic research that used as an essential method for obtaining information the field research in a population of 229 teachers, of which 83 were the sample. This research was carried out from September 2016 to October 2017. The utilization of ethnographic techniques complemented the methodology to achieve as a main outcome a cultural-pedagogical-operative matrix for the educational practice in the teaching of mathematics and, at the same time, revealed theoretical limitations in the understanding of the scope of the pedagogical management of teachers in the acting model in Santiago de Cuba. This result creates the needed methodological conditions for the transfer of good practices in the learning of mathematics supported by ethnography also opens a space for reflection and participation in the way of managing the learning process.
\end{abstract}

Key words: teachers, mathematics education, thinking, ethnography.

\footnotetext{
1 Profesor adjunto de la Universidad de Oriente, Subdirector General Provincial de Santiago de Cuba. Doctor en Ciencias Pedagógicas. ORCID https://orcid.org/0000-0001-7293-2737 Dirección electrónica: Osmanyjustiskatt@yahoo.com

2 Profesora del Instituto Preuniversitario Urbano Antonio Santiago del Municipio Contramaestre en la provincia Santiago de Cuba. Licenciada en Educación en la Especialidad de Matemática. Dirección electrónica:kmelia85@nauta.cu
}

Artículo recibido: 23 de marzo, 2019

Enviado a corrección: 17 de junio, 2019

Aprobado: 19 de agosto, 2019 


\section{Introducción}

El preuniversitario es el último nivel educativo del sistema general en Cuba, sus egresados van al trabajo o a la Universidad para formarse como profesionales. El acceso a los estudios de la Educación Superior está determinado por la realización de exámenes de ingreso en las asignaturas de Historia de Cuba, Español Literatura y Matemática. Esta última ha sido la materia en la que el estudiantado muestra bajos desempeños. Por ello, esta investigación se centra en buscar respuestas y crear condiciones pedagógicas y epistemológicas para el perfeccionamiento de la enseñanza de la matemática en el Sistema Nacional de Educación a partir del estudio del pensamiento profesional pedagógico del profesorado de matemática.

El objetivo de este trabajo es realizar un acercamiento etnográfico a la forma en que el profesorado de matemática concibe el diseño de su gestión pedagógica para formar conocimientos duraderos en el alumnado. Como antecedentes de este trabajo se han realizado, desde el 2011 a la fecha, tres estudios de profundización por especialistas del Instituto de Central de Ciencias Pedagógicas, el Ministerio de Educación en Cuba, la Dirección Provincial y las universidades Frank País y Oriente. Dichos antecedentes respondieron a la necesidad de buscar causas de los resultados que, en los exámenes de ingreso a la Educación Superior, se han venido obteniendo. Estos esfuerzos han aportado niveles de solución, pero no han sido suficientes, pues no solo se trata de preparación en la didáctica y el contenido de la asignatura, sino que también es cuestión de la competencia cultural profesional pedagógica propia del contexto santiaguero.

En el trabajo de campo surgió la necesidad de profundizar en el pensamiento del profesorado como matriz cultural en la que se generan las prácticas docentes para la enseñanza de la Matemática. Las soluciones a los problemas que tradicionalmente han surgido en el aprendizaje y la enseñanza se han creado en diversas aristas: unas en la dimensión didáctica, otras en las estrategias de aprendizaje, la dirección del aprendizaje y el dominio por el profesorado de los contenidos de la matemática. En esta investigación se realizó un acercamiento a partir de otra dimensión inédita en investigaciones en el contexto de Santiago de Cuba: el pensamiento del profesorado.

La utilidad de realizar estudios etnográficos del pensamiento del profesorado es evidente si se tiene una posición participativa y realista del proceso educativo en las aulas. La existencia de currículos pertinentes, condiciones materiales en las escuelas, resultados de investigaciones en la didáctica y saberes de las ciencias matemáticas no son suficientes 
si el profesorado que imparte la asignatura no incorpora en su pensamiento profesional pedagógico estos conocimientos de forma consciente. En la relación del saber, el deber hacer, el querer hacer y el hacer, la descripción densa del pensamiento del profesorado constituye un universo al que se le presta atención para situar la dimensión humana como aspecto determinante en el logro de aprendizajes de calidad en la enseñanza de la matemática.

\section{Referente teórico}

La estructuración de la investigación parte de considerar como referente el enfoque del pensamiento del profesorado surgido en el campo de las investigaciones pedagógicas en el segundo lustro de la década de los años 60 del siglo XX. En trabajos sistematizados por Martínez (1988) se recorre históricamente la evolución y objeto de este enfoque, el cual se centra en el profesorado como sujeto mediador y ofrece grandes posibilidades para comprender lo que piensa como solución a los problemas que surgen en el proceso de enseñanza aprendizaje. El pensamiento pedagógico del profesorado como enfoque ha ido desarrollándose desde el año 1968 en estudios realizados por Barquín Ruiz (1991); Martínez (1988); Serrano (2010); Soto, Juárez y García (2011), y Zamora y Balmaceda (2015), siempre en torno a tres cuestiones esenciales: el estudio de las percepciones del profesorado con respecto a las actitudes del alumnado, la reflexión sobre las actividades del profesorado en el aula y la solución que da a problemas didácticos.

Estas tres direcciones han tenido un punto de encuentro en el estudio de los modos en que el conocimiento es asimilado y utilizado por el profesorado, y las circunstancias que afectan su adquisición y empleo. La preocupación principal de este enfoque teórico ha estado en describir los procesos de razonamiento del profesorado durante su actividad profesional. Su uso actual, según Justis (2013), permite trascender su origen cognitivista, constructivista o su ajuste a la teoría práctica de la educación y llegar a realizar contribuciones para la transformación de la práctica educacional y la comprensión de la cultura profesional del profesorado que se relaciona con la toma de decisiones pedagógicas en contextos culturales específicos.

Para el estudio del pensamiento del profesorado se ha asumido como cuestiones importantes: el análisis de atribuciones, el peso de la experiencia en las actitudes, el intercambio de saberes culturales entre las personas y la presión institucional contenida en las normas de comportamiento. El común denominador de todos los estudios ha estado 
orientado a profundizar en la postura del profesorado ante su responsabilidad con los logros de aprendizaje del alumnado y las decisiones derivadas de su práctica profesional para el cumplimiento de dicho objetivo y el tratamiento a los resultados educativos.

A partir de los estudios de Serrano (2010), con relación a las creencias y a las concepciones de las personas, se estructura la matriz que sirve de marco de referencia para la acción en el pensamiento del profesorado. Por creencias se precisa en este trabajo, a partir de la sistematización realizada de la obra de Serrano (2010), a los preconceptos, conocimientos subjetivos basados en la experiencia y los sentimientos no fundamentados en la ciencia pedagógica que posee el profesorado, y que codifica y justifica su uso en la práctica personal áulica. En el contexto santiaguero se registra en la frase: cada docente tiene su librito $^{3}$. Por otra parte, se define operacionalmente para esta investigación, la concepción como aquellos organizadores implícitos de conceptos y categorías de naturaleza cognitiva fundamentados en las ciencias pedagógicas y que influyen sobre las creencias (Serrano 2010).

En esta perspectiva, el pensamiento del profesorado se asume como un constructo que tiene referentes en Berger y Luckmann (1991) y da cuenta de la construcción social de la realidad escolar concreta en la tendencia fenomenológica docente y sistematiza aspectos abordados por Justis (2013) al considerar los procesos subjetivos como realidades objetivadas culturalmente. El profesorado experimenta la vida cotidiana en la escuela como una realidad ordenada y objetivada por las relaciones sociales en las que se desarrolla y construye la cultura escolar. Constituyó, también, un referente en el desarrollo de esta investigación, la teoría de las representaciones sociales. La sistematización realizada por Araya (2002) señala a Jodelet (1984) y Moscovici (1979, 1981, 1984, y 1991) para definir representación como la acción de construir en el pensamiento un equivalente mediado por una figura. Las representaciones sociales hacen referencia a un tipo de conocimiento que describe cómo la gente piensa y organiza su vida cotidiana a través de una matriz denominada popularmente como conocimiento del sentido común.

El sentido común es, según Araya (2002), una forma de percibir, razonar y actuar pautada por conocimientos sociales sistematizados como experiencias. Estos conocimientos incluyen contenidos cognitivos, afectivos y simbólicos que tienen una función orientadora,

${ }^{3}$ En adelante se escribirá en cursiva y sin comillas fases o palabras registradas en el trabajo de campo como expresión de la manera en que el profesorado concibe, denomina y atribuye significados a su práctica. Los autores, respetarán literalmente estas expresiones para someterlas a interpretación crítica y argumentativa del modelo actuante. (Nota de los autores) 
organizativa y comunicativa. Además, son sistemas cognitivos portadores de estereotipos, opiniones, creencias, valores y normas que pueden tener impacto positivo o negativo en la gestión de aprendizaje en la labor pedagógica. De los anteriores referentes, surge la categoría pensamiento profesional pedagógico, aportada por Justis (2013), en que se realiza la vida profesional docente en el contexto de la cultura escolar. De su constitución situacional y contextualizada se determinan las matrices culturales y las experiencias concretas con las que se enfrenta a diario el profesorado en la solución de los problemas de formación del alumnado y, al mismo tiempo, se erigen su identidad laboral y las formas culturales en que perciben la realidad escolar.

La descripción densa de la actividad profesional del profesorado de matemática realizada en la investigación parte de los fundamentos de la obra de Geertz (1973), contextualizada en los estudios compilados por Bohannan y Glazer (2003), que la define como la observación de fenómenos para la constatación del hecho cultural en el contexto de actuación del sujeto. Requiere desentrañar su trasfondo, ahondar para especificar su intencionalidad y naturaleza, observar lo indiscutible de forma externa y profundizar, describir el hecho para revelar una intención en la búsqueda de su naturaleza o esencia.

Otro de los fundamentos etnográficos fue la perspectiva multi situada, contextualizada en el trabajo de campo en la provincia Santiago de Cuba con un carácter des-localizado, sustentado en los estudios de Escobar (2005) que enfoca la generación de posiciones profesionales reflexivas, unido al registro de la especificidad de las creencias y concepciones propias del profesional de matemática en el contexto de Santiago de Cuba en el escenario de la escuela media. De este fundamento se derivó un procedimiento que favoreció la discusión de validez y pertinencia para la cultura escolar y las pretensiones formativas del magisterio en las condiciones concretas de cada grupo humano implicado. El uso de la etnografía multi situada en el estudio del pensamiento del profesorado de matemática dio cuenta, en esta investigación, de una perspectiva metodológica en la cual resulta insuficiente la seguridad y validez del trabajo de campo limitado a una situación o forma de pensar local como garantía de veracidad del conocimiento profundo del pensamiento del profesorado.

Con la perspectiva multi situada, en esta investigación se sistematiza los resultados de Walf (1982), Comaroff y Comaroff (2003) en que el espacio (aula, escuela, nivel educativo y/o municipio) no es solo sitio específico, sino construcción social y política, históricamente situada y en estrecha relación con los procesos nacionales y globales. En la descripción etnográfica se utilizan términos con los cuáles son analizadas las creencias y concepciones 
del profesorado como códigos construidos y usados en diversos contextos de producción de sentidos y significados, asociados a la formación de conocimientos matemáticos que favorecen la comprensión profunda del pensamiento profesional pedagógico.

La pretensión del artículo no alcanza la valoración de pertinencia del pensamiento profesional pedagógico del profesorado de matemática, se ciñe en crear una fuente para la reflexión y la toma de decisiones por los propios actores sociales en el plano individual y grupal. Además, de brindar a los investigadores elementos fácticos de la cultura escolar para la generación de propuestas y la socialización de buenas prácticas que contribuyan al perfeccionamiento de la enseñanza de la matemática en la provincia.

La perspectiva etnográfica multi situada permite trascender el trabajo de campo de un municipio o escuela específica, como los lugares y situaciones locales de la investigación etnográfica convencional, para crear las condiciones analíticas que favorecen una reflexión profunda de la cual se pueden identificar y transferir buenas prácticas a otros contextos culturales y/o eliminar creencias o concepciones no pertinentes a los fines formativos.

El uso de la perspectiva antes abordada permitió examinar la circulación de significados, objetos e identidades culturales para llamar la atención sobre la expresión universal de lo singular y ubicar el estudio de la relación del etnógrafo y el profesorado de matemática como una interacción productora de soluciones en la gestión de aprendizaje. Su empleo en la investigación supuso una estrategia metodológica en la que resultó necesario involucrar más de una localidad, territorio o lugar para llevar adelante el proceso de gestión de aprendizaje. En el plano metodológico ayudó a derivar de la tradicional relación emic-etic una propuesta sistematizada por Justis, Almestro y Silva (2017) contextualizada en la dinámica del modelo percibido y el modelo operatorio en la interacción del profesorado de matemática y el etnógrafo.

Dentro de los referentes, se sistematiza la perspectiva defendida por Justis (2013), la cual sustenta la cultura escolar como matriz pedagógica-metodológica-operacional y la concibe como una herramienta gnoseológica construida por grupos sociales y generaciones en su labor pedagógica, expresada en las relaciones: educación, formación, diagnóstico y participación del grupo de docentes en la toma de decisiones. Esta perspectiva teórica permitió re-significar y redimensionar la cultura escolar como categoría pedagógica para comprender los elementos culturales con los que concibe el profesorado de matemática el diseño de su actuación, lo que la convierte en actividad referencial en cualquiera de los contextos en que, de forma intencional, influya sobre la personalidad del alumnado para la 
formación de conocimientos. La cultura escolar condensa la representación pedagógica de cómo debe ser la actuación del profesorado para lograr aprendizaje contextualizado a las condiciones concretas en que se realiza el proceso de aprendizaje. Su estudio crítico y reflexivo ayuda a una mejor comprensión de la realidad escolar, lo que la convierte en una herramienta para generar aprendizajes significativos.

La pertinencia pedagógica de la cultura escolar en el estudio del pensamiento del profesorado crece al revelar procesos que deben ser formalizados en la observación, interpretación y comprensión desde las ciencias pedagógicas y, en un sentido práctico, orienta a tomar decisiones enfocadas en la reflexión de su propia práctica y experiencias docentes. Al concebir al profesorado de matemática como productor de sentidos, el estudio etnográfico identifica las construcciones culturales simbólicas en los significados y en el lenguaje a través de los cuales construyen el mundo laboral en que educan al alumnado y se forman como profesionales.

La labor pedagógica del profesorado de matemática tiene, en la relación pensamientoacción, un núcleo importante de análisis, el cual se sintetiza en la categoría gestión de aprendizaje, que parte de considerar que la actividad pedagógica que desarrolla el profesorado de matemática es, no solo un proceso didáctico que combina conocimientos y metodología para su enseñanza, sino que en ella, también se codifica el diseño de la ayuda pedagógica como un proceso de orientación, dirección y asesoramiento de la educación para la formación de conocimientos matemáticos.

Teniendo en cuenta la sistematización realizada por Justis et al., (2017), en este trabajo se entiende por gestión de aprendizaje, en el nivel áulico, la organización del proceso pedagógico y didáctico de búsqueda por el alumnado de información, el estudio de alternativas y la experimentación de soluciones. Se basa en la relación de primer orden profesorado-alumnado y de segundo orden alumnado-alumnado y profesorado-alumnadofamilia. En estas relaciones, la gestión de aprendizaje se concreta como la creación e implementación de herramientas que garanticen la retención, la comprensión, el uso sistemático y útil del contenido en el proceso de enseñanza y de aprendizaje en relación con la vida práctica de los implicados.

Gestionar aprendizajes en la labor pedagógica del profesorado de matemática comprende, según estudios de Justis (2019), a) elaborar y comprometer en la solución de planes correctivos, b) realizar despachos pedagógicos, c) establecer estrategias 
compensatorias, d) monitorear las rutas de aprendizaje del alumnado a través de los métodos de diagnóstico y de matrices de aprendizaje.

Las herramientas que esta investigación pone en manos de profesionales de la docencia para la realización de la gestión de aprendizajes son: a) el equipamiento tecnológico disponible en las escuelas, b) el uso del método etno-analítico matricial sistematizado por Justis, (2013) y c) los procedimientos de ayuda pedagógica para la realización de una pedagogía diferencial en el proceso de enseñanza y de aprendizaje.

La gestión del aprendizaje matemático tiene como base la integración de lo contextual, lo pedagógico y lo psicológico en el que el concepto aportado por Kurt Lewin ${ }^{4}$ de espacio vital ocupa un lugar explicativo importante al hacer referencia a todo lo que necesita saber una persona para comprender su conducta concreta en un ambiente psicológico específico en un tiempo dado y que enfatiza en lo cognitivo: sensación, percepción, imaginación, recuerdo y pensamiento. Resulta, también importante, tener en cuenta, que el alumnado aprende por medio de la generalización, la diferenciación y la reestructuración de su personalidad y de su ambiente psicológico, aspecto que argumenta la valía del fundamento asumido de la teoría de Kurt Lewin para la gestión de aprendizaje.

El aprendizaje debe ser visto, en su gestión, según Justis et al. (2017) como un accionar del propio alumno en un contexto cultural específico que propicia, al proceso docente, partir de situaciones que se presentan en la realidad el material fáctico necesario para reflexionar sobre su propia existencia. Un problema real convertido en necesidad, hace del proceso de búsqueda de respuestas y soluciones un aprendizaje significativo y duradero que potencialmente modela las emociones y los sentimientos para fomentar los valores que conducen a elevar el nivel cognitivo a partir de la tarea que aporta soluciones.

La tarea docente es otro aspecto que desempeña un importante papel en la gestión de aprendizaje. Su diseño en el proceso docente parte de conocimientos empíricamente construidos por el sujeto en su vida cotidiana, se basa en las diferencias y semejanzas entre los fenómenos dados generalmente por la observación y comparación en niveles de abstracción cada vez superiores. A este conocimiento empírico debe integrarse el conocimiento teórico, que se construye por medio de métodos indagatorios en la resolución de las contradicciones en un área problemática central en que se desarrollan ideas sobre los orígenes, relaciones y dinámica de los fenómenos y, se comunica por medio de modelos. La

\footnotetext{
${ }^{4}$ Kurt Lewin (1890 - 1947) figura clave en la teoría cognitivista del aprendizaje enfocó sus estudios no tanto al aprendizaje sino a la motivación y la percepción. (Nota de los Autores)
} 
gestión de aprendizaje debe facilitar acciones, por el profesorado, para que el alumnado haga converger lo empírico y lo teórico en la solución de las tareas docentes de manera integrada.

Esta posición metodológica es importante pues crea en el alumnado una lógica entre conocimientos y comportamientos, entre lo teórico y lo procedimental, para favorecer el cambio en el significado de la experiencia social e individual. Con esta visión se genera un proceso pedagógico que está continuamente incrementando su capacidad para producir resultados sobre lo que realmente le preocupa como sociedad al alumnado y al magisterio.

El centro de la gestión de aprendizaje es el alumnado y se trata de que esté en cada momento, bajo la conducción docente, descubra buenas prácticas y las incorpore a sus estrategias de aprendizaje.

\section{Metodología}

\subsection{Enfoque}

La investigación se realizó con enfoque cualitativo y es de tipo etnográfica. El alcance del proceso de investigación concreta una descripción densa del pensamiento pedagógico profesional del profesorado de matemática condicionante de una reflexión crítica de su modo de actuación y en consecuencia la adopción de decisiones que generan el perfeccionamiento del proceso de gestión de aprendizaje.

\subsection{Población del estudio}

Esta investigación se desarrolló desde septiembre 2016 hasta octubre 2017, se tomaron como muestra 83 personas del claustro de matemática que trabajan en 32 de los 44 preuniversitarios de la provincia Santiago de Cuba en sus nueve municipios que representan el $72,7 \%$ de las instituciones y el 36,2\% del profesorado de una población docente de 229 que imparten la asignatura.

La selección de la muestra fue de manera intencionada y con el consentimiento de las personas implicadas lo cual favoreció el acceso y validación de los datos, así como la participación activa en el proceso de recogida e interpretación de los datos cualitativos en el trabajo de campo realizado. Resultó una premisa favorable para lograr el acceso de del equipo investigador al trabajo de campo el hecho de que eran parte del grupo objeto de estudio, uno como miembro permanente del profesorado y otro como parte de las estructuras metodológicas y asesoras encargadas de la preparación del profesorado. 
Con el propósito de crear condiciones para obtener información amplia se intencionó, en la selección de la muestra, a una parte del profesorado con altos resultados (43) y el resto (40) con resultados entre bajos y medios, siempre garantizando la presencia de docentes de los nueve municipios de la provincia.

El método de selección de la muestra fue intencional y se realizó con el profesorado que trabaja en el duodécimo grado por constituir este grado el que cierra el Sistema Educativo General en Cuba y el que culmina la preparación de la población estudiantil que aspira a acceder a la universidad en alguna de sus carreras de nivel superior. En este grado, por tradición, en el Sistema Educativo Cubano, se realizan acciones intensivas de preparación al alumnado en los contenidos matemáticos de los diferentes niveles educativos que, en criterio de la universidad, son básicos para garantizar calidad en el ingreso a las diferentes carreras.

\subsection{Técnicas de recolección}

En el desarrollo del método de trabajo de campo se emplearon las técnicas etnográficas de: observación y entrevista semi-estructuradas participantes, grupos focales, diario campo, cuestionario, la historia de vida profesional y el grupo de discusión para obtener la información en torno a cómo se representa en el profesorado de matemática la gestión pedagógica para lograr aprendizajes de calidad en sus estudiantes.

El empleo de las técnicas de grupo focal y grupo de discusión fue siguiendo criterios de Krueger (1991) y Callejo (2001) quienes diferencian la finalidad de ambas técnicas, la primera para obtener y profundizar en los significados de la información inicial obtenida y la segunda para valorar el impacto de acciones realizadas. Ambas técnicas fueron aplicadas a 23 personas de las 83 que constituyeron la muestra, 16 de ellas participaron como fuentes en las dos técnicas.

El grupo focal fue usado para obtener información y profundizar en campos temáticos como estrategias para la atención a la diversidad de desempeños cognitivos de los estudiantes, identificación de ritmos de aprendizaje, prácticas en el uso de los libros de textos mientras que la técnica grupo de discusión se empleó para conocer las valoraciones de los participantes del impacto de las estrategias identificadas y transferidas en el trabajo de campo al grupo de docentes en el proceso reflexivo que implicó el empleo de la etnografía. El resto de las técnicas fueron utilizadas para reunir información suficiente y realizar triangulaciones que al llegar al grupo focal eran confirmadas, ampliadas y rectificadas. 
En la elaboración del registro etnográfico e interpretación de la información se empleó, en relación dialéctica, el método de triangulación de información y el registro de caso único, bajo el criterio de que una forma de pensar que coincide en varios miembros del profesorado tiene tanta importancia, como información fáctica, que una en la que no hay grandes niveles de coincidencia. Por ello, se registra en forma de descripción densa toda la información obtenida sin distinción y con criterio inclusivo sin importar las proporciones cuantitativas por lo que los datos registrados e interpretados son de tipo cualitativo.

El trabajo de campo en general se orientó en la aplicación de las diferentes técnicas etnográficas siguiendo un cuestionario genérico que dentro de sus contenidos precisó:

1. ¿Quiénes están en el grupo? ¿Cuántos son y cuáles son sus tipos, identidades, y características relevantes para aprender? ¿Cómo se consigue ser miembro del grupo?

2. ¿Qué hacen los individuos del grupo? ¿Qué se dicen entre sí de las estrategias de aprendizaje?

a) ¿Qué comportamientos son repetitivos y cuáles anómalos? ¿En qué acontecimientos, actividades o rutinas están implicados, los individuos como parte de la gestión de aprendizaje? ¿Qué recursos se emplean en dicha actividad? ¿Cómo son asignados? ¿Qué contextos diferentes es posible identificar?

b) ¿Cómo se comportan las personas del grupo recíprocamente? ¿Cuál es la naturaleza de la participación y de la interacción? ¿Cómo se relacionan las personas y cómo se vinculan? ¿Qué status y roles aparecen en su interacción? ¿Quién toma las decisiones y por quién? ¿Qué organización subyace a todas estas interacciones?

c) ¿Cuál es el contenido de sus conversaciones? ¿Qué temas son comunes? ¿Cuáles poco frecuentes? ¿Qué relatos, creencias, activas, sonoras y auditivas, y sentimientos se detectan en los contextos del grupo?

3. ¿Cuándo se reúne el grupo para gestionar aprendizaje? ¿Con qué frecuencia se producen las reuniones para gestionar aprendizaje? ¿Cuánto se prolongan? ¿De qué modo el grupo conceptualiza, emplea y distribuye el tiempo para gestionar aprendizaje? ¿Cómo perciben los participantes su pasado, su futuro dentro de este grupo?

4. ¿Cómo se interrelacionan los elementos identificados, desde el punto de vista del participante y desde el punto de vista del investigador? ¿Cómo se mantiene la estabilidad en el grupo? ¿Cómo surge y es orientado el cambio para gestionar 
aprendizaje? ¿Cómo son organizados todos los elementos determinados para gestionar aprendizaje? ¿Qué reglas, normas y costumbres rigen en la organización para gestionar aprendizaje? ¿Cómo se relaciona este grupo con otros grupos y organizaciones?

5. ¿Por qué funciona el grupo como lo hace? ¿Qué significados atribuyen los actores sociales a su conducta en la gestión del aprendizaje? ¿Qué símbolos, valores y concepciones del mudo se pueden descubrir en él?

Como parte de la validación y coherencia de la descripción etnográfica se puso en manos del profesorado que fue parte de la muestra el resultado del trabajo de campo para que completaran la información o emitieran los desacuerdos que consideraban no se ajustaban a las formas de pensar actuante en el modelo percibido. Luego de esta triangulación entre profesorado de matemática, texto etnográfico y etnógrafo se volvió a la interpretación y redacción del informe etnográfico.

\subsection{Procesamiento de análisis}

Tras la aplicación de la técnicas de observación y entrevista semi-estructuradas y su comparación con los elementos descritos en el diario de campo elaborado se realizaron aproximaciones de profundización progresiva y longitudinal desde la creación por los actores sociales (profesorado de matemática) de historias de vida profesional y autobiografías que permitieron la conformación de un texto descriptivo inicial que luego fue eje del debate, apoyados en la técnica grupo de discusión con la cual, no solo se logró la descripción del modelo percibido de la población objeto de estudio, sino que, se ganó consentimiento del contenido de la descripción de actividades, sentidos y significados registrados en el texto etnográfico resultante del trabajo de campo. Este proceso favoreció el logro de la sensibilización de las personas implicadas y la inducción a gestar cambios en el modo de actuación para la gestión de aprendizaje en la asignatura de matemática.

Al triangular la información obtenida y con la participación del profesorado se organizó un texto etnográfico que estructura unidades de significados en categorías en las que se identifican campos temáticos que expresan la relación entre creencias y concepciones que desde el punto de vista epistemológico devino en una relación metodológica entre el modelo percibido (la visión del profesorado) y el modelo operatorio (la sistematización teórica en la visión del investigador). 
Este proceso se realizó asumiendo como criterios el registro de toda la diversidad de ideas en la descripción sin que se ponderara aquellos en que más personas coincidían; la libertad de tomar posición ante cada aspecto constitutivo del texto etnográfico y de realizar propuestas de modificaciones para acendrar su verosímilidad y la comparación de los aspectos de la descripción con la teoría de las ciencias de la educación que los refutan o justifican.

El resultado de la triangulación también devino en una categorización en la que como resultado de la aplicación del grupo focal primero y del grupo de discusión después, se conceptualizaron las estrategias directrices para la gestión de aprendizaje por el profesorado de matemática.

Se definió como estrategias directrices a las actividades más estables en el comportamiento docente con buenos resultados y a las cuales estos atribuyen la razón principal que justifican el éxito en el proceso de enseñanza y de aprendizaje en la preparación para el ingreso a la Educación Superior. Se denominan directrices porque marcan pautas en la cultura profesional y escolar para derivar de ellas las acciones y tareas necesarias para cada estudiante en correspondencia al contexto propio en el que vive.

Finalmente como parte del procesamiento de la información se establece una matriz cultural-pedagógica-operatoria que, desde el punto de vista teórico, establece relaciones dialéctica entre modelos percibidos y modelos operatorios resultante de la relación entre creencias y concepciones de la forma de realizar la actividad pedagógica en el aprendizaje de la matemática; y de forma práctica, orienta la lógica reflexiva que conduce a la determinación de las estrategias, las acciones y las tareas más pertinentes para en el contextos cultural específico de cada escuela organizar el proceso de gestión de aprendizaje.

Para la interpretación del registro etnográfico en este informe se distingue las frases textuales recogidas en el trabajo de campo en letra cursiva y cuando lo requiere, se profundiza en sus significados y sentidos. También se utilizan los referentes teóricos antes tratados en el artículo y que metodológicamente son sintetizados como modelo operatorio. En relación dialéctica y como síntesis de la relación ser-deber ser, realidad escolar-realidad deseada se conceptualiza la visión actual docente como modelo percibido. 


\section{Resultados}

Como resultado del trabajo de campo y de la descripción etnográfica realizada se apreciaron prácticas, se profundizó en significados y creencias hasta lograr reconstruir experiencias que una vez fundamentadas devinieron en estrategias directrices que se convierten en categorías que sintetizan las buenas prácticas para la solución del problema que inicialmente motivó esta investigación.

En el orden metodológico, en la práctica educativa santiaguera se han venido sistematizando seis estrategias directrices de gestión pedagógica que se estructuran en un sistema y conforman un marco general de actuación fruto del consenso de qué es lo que se debe hacer para lograr aprendizajes duraderos en el alumnado.

Las estrategias directrices que se sistematizan como buenas prácticas son:

1) Impartición de clases de calidad. 2) Atención a las diferencias individuales. 3) Preparación permanente del profesorado para resolver problemas de aprendizajes. 4) Desarrollo de estrategias de aprendizajes en el alumnado. 5) Sistematización de contenidos. 6) El sistema de evaluación sistemática de resultados.

En la modelación de las clases se hicieron precisiones en el trabajo de campo a la tipología de entrenamiento o sistematización para el nivel preuniversitario para ello se diferenciaron tres tipos de influencias:

- La clase para el cumplimiento del programa del currículo general que, se caracteriza por un agrupamiento estable del alumnado denominados grupos docentes y que tienen que cumplir con el programa de estudio en un tiempo institucionalmente determinado.

- La clase de sistematización que, en horarios establecidos por cada escuela en su currículo institucional agrupa de manera flexible a una población estudiantil de diferentes grupos docentes y que presentan dificultades en un mismo objetivo, componente o elemento del conocimiento.

- La clase de atención diferenciada o remedial que, se desarrolla con pequeños grupos estudiantiles, intencionalmente conformados por el claustro, que requieren de mayor nivel de ayuda y que tienen mayores problemas de aprendizaje.

Para el desarrollo de la estrategia de atención a las diferencias individuales se han enfocado cinco actividades principales de realización:

1) El horario: espacio para organizar la atención a diferencias individuales. 
2) La preparación metodológica: espacio de aseguramiento didáctico para la atención a diferencias individuales.

3) La clase: espacio de realización de la atención a diferencias individuales.

4) El sistema de dirección como espacio para el monitoreo y proyección de las tomas de decisiones pedagógicas.

5) En la evaluación sistemática como espacio de medición de impacto de la atención a las diferencias individuales.

La preparación metodológica como estrategia se modeló acorde a las necesidades particulares de cada miembro del claustro y las características culturales de cada institución escolar. En ellas se sistematizaron acciones como:

- La preparación metodológica concentrada del profesorado de un mismo grado y de diferentes escuelas.

- La preparación metodológica diferenciada a miembros del claustro de una misma asignatura y de cualquier grado que presenta bajo desempeño en contenidos determinados.

- La dedicación de la mayor cantidad del tiempo de la preparación metodológica a la discusión colectiva del sistema o subsistema de clases de cada miembro del profesorado para la socialización de experiencias y alternativas de desarrollo de los contenidos.

Para el desarrollo de estrategias de aprendizaje no solo se ha dado preparación al profesorado para su orientación y desarrollo sino, que se han registrado las buenas prácticas observadas en el alumnado con altos resultados. El registro de las buenas prácticas ha sido socializado en un epistolario en el que se sintetizan recomendaciones y describen estrategias para el aprendizaje de los contenidos en que históricamente se registran bajos resultados.

Para la sistematización de los contenidos se desataca una de sus formas denominada informalmente por el profesorado como trabajo con temarios y que se basa en tres formas fundamentales de trabajo:

1. Como instrumento de medición de aprendizaje: se refiere a la medición en un instrumento de preferencia utilizado en cursos anteriores como pruebas finales del 
nivel (sexto, noveno y duodécimo grados) que permiten al profesorado actualizar el diagnóstico y al alumnado el empleo de sus conocimientos de forma sistemática.

2. Como herramienta para el desarrollo de las clases de sistematización: se caracteriza por la conformación de un temario en el que se agota todas las formas de preguntar en que un contenido puede ser medido para evidenciar la calidad del cumplimiento de un objetivo utilizando todas las vías de solución y posibles respuestas.

3. Como forma de trabajo independiente: es la selección de un temario, para el tratamiento a las necesidades cognoscitivas personalizadas a miembros del grupo estudiantil, que debe ser resuelto de forma individual en un plazo de tiempo determinado con la aplicación por el profesorado de niveles de ayuda.

Para el sistema de evaluación se desarrollan los cortes preliminares que han permitido la valoración en tiempo real del cumplimiento de los objetivos del programa de estudio organizado en sistemas y subsistemas de contenidos lo que ha permitido la toma de decisiones pedagógicas en tiempo real para la corrección de los problemas de aprendizaje manifiestos en el proceso pedagógico.

En la práctica pedagógica en las escuelas en no pocas ocasiones se observa una tendencia a sobredimensionar la cantidad de contenidos impartidos como expresión de cumplimiento de programas de estudio, sobre la calidad de los conocimientos adquiridos en el desarrollo del programa. Para redimensionar esta desviación del curso de la acción pedagógica pertinente se establecen ciclos de gestión de buenas prácticas como una herramienta que favorece la labor pedagógica del profesorado y a la vez su autorreflexión en el perfeccionamiento del proceso de gestión de aprendizaje.

Estas estrategias directrices conceptualizadas como resultado del trabajo de campo a partir de las buenas prácticas registradas fueron estudiadas por el claustro de la provincia e implementadas contextualizándolas a las condiciones culturales, educativas y materiales de cada grupo estudiantil y del profesorado. Solo se solicitó a los implicados que si entendía necesarias las estrategias le diseñaran acciones a cada una de ellas para conformar un modo de gestionar los aprendizajes. También se les explicó que no debía limitarse a usar solo estas estrategias, sino que ellos, a partir de sus experiencias y el diagnóstico de cada grupo estudiantil y las características del lugar en que estaba situada la escuela podían crear otras estrategias y fundamentarlas. 
El desarrollo de la investigación no tuvo un alcance solo descriptivo, de manera implícita y como experiencia de campo, se fundamenta una herramienta pedagógica para convertir la reflexión crítica de la realidad escolar y en ella la actuación de cada docente para lograr aprendizajes de calidad en sus estudiantes. Esta herramienta surge de un modelo genérico (ver figura 1) con el cual se estructura una lógica reflexiva de creencias y concepciones hasta lograr un consenso, determinar estrategias pertinentes y derivar acciones y tareas que aseguren su realización.

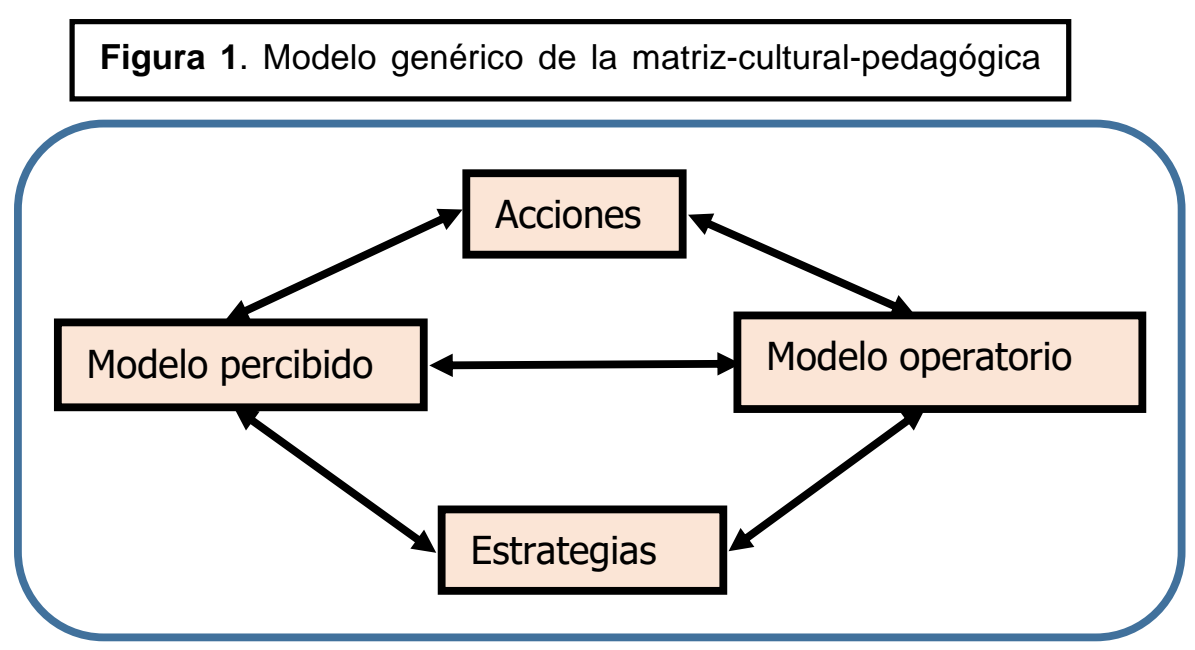

Fuente: Elaboración propia de los autores (2019)

De la relación dialéctica entre el modelo percibido y el modelo operatorio como resultante del trabajo de campo realizado en el que el grupo de docentes que fue muestra de la investigación en el proceso de generación, registro, consenso y socialización de la información llega a acuerdos de cuáles son las estrategias directrices que serán las que caracterizarán la gestión de aprendizaje del profesorado.

En el proceso de investigación etnográfica se llega al consenso de estrategias a partir del empleo de las técnicas grupo focal primero y de discusión luego de manera que el proceso de conformación del resultado etnográfico trasciende epistemológicamente sus características descriptivas e interpretativas para mejorar la comprensión y convertirse en un proceso reflexivo y de toma de decisión pedagógica por los participantes quienes luego de un proceso de autorreflexión y debate identifica en sus prácticas aquellas formas de hacer que en la experiencia y en la ciencia son necesarias sistematizar en el contexto de la cultura escolar en que se desarrolla el proceso pedagógico. 
Esta herramienta se orienta en cuatro componentes identificados en: 1) Autoetnografía del pensamiento del profesorado: descripción densa de lo que hago ¿qué es lo que hago para el desarrollo de esa actividad? (Conformación de descriptores y registros etnográfico). 2) Manejo simbólico ¿qué significa lo que hago? Se refuerzan puntos de vistas, principios y creencias de la mejor manera de desarrollar una actividad pedagógica. (Conformación de criterios de realización). 3) Historia de vida profesional ¿cómo he llegado a ser así? (Conformación de memorias de soluciones de problemas situacionales) 4) Nueva visión de la realización de la actividad ¿cómo podría hacer las cosas diferentes para alcanzar mejores resultados? (Conformación del marco de la nueva gestión). Los tres primeros componentes conforman el modelo percibido y el cuarto el modelo operatorio que dinamiza la forma en que se realiza la actividad pedagógica.

Para el registro de las determinaciones derivadas de la reflexión crítica de la práctica pedagógica en la que se anteponen creencias y concepciones se propone la matriz culturalpedagógica-operatoria que desde el punto de vista práctico sintetiza la toma de decisiones fruto del proceso de análisis realizado por los implicados en el proceso de investigación. La tabla 1 de doble entrada, permite ordenar el registro de las decisiones es contentiva de la derivación e implementación de las estrategias, acciones y tareas necesarias para la realización práctica de las determinaciones surgidas de las creencias y concepciones.

La determinación de las estrategias directrices como resultante de la reflexión primero individual y luego colectiva tiene en la organización de la matriz cultural-pedagógicaoperatoria un espacio de contextualización e implementación en cada participante en el que una vez que han quedado establecidas las estrategias directrices cada docente a partir de las características propias y de sus estudiantes y en correspondencia con los medios tecnológicos y escolares disponibles crea las acciones y tareas que aseguran la realización de las estrategias acordadas. 
Tabla 1 Matriz cultural pedagógica operatoria

\begin{tabular}{|l|l|l|}
\hline Estrategias matrices & Acciones & \multicolumn{1}{|c|}{ Tareas } \\
\hline La calidad de las clases & & \\
\cline { 2 - 3 } & & \\
\hline $\begin{array}{l}\text { La atención a las } \\
\text { diferencias individuales }\end{array}$ & & \\
\cline { 2 - 3 } $\begin{array}{l}\text { La sistematización de } \\
\text { contenidos }\end{array}$ & & \\
\hline $\begin{array}{l}\text { Desarrollo de estrategias de } \\
\text { aprendizajes }\end{array}$ & & \\
\cline { 2 - 3 } $\begin{array}{l}\text { La preparación } \\
\text { metodológica del docente }\end{array}$ & & \\
\hline \multirow{2}{*}{ El sistema de evaluación } & & \\
\cline { 2 - 3 } & & \\
\hline
\end{tabular}

Fuente: Elaboración propia de los autores (2019)

Las preguntas genéricas con la cuales se estructuraron las diferentes técnicas etnográficas utilizadas y de las cuáles se conforma la propuesta fue: ¿Por qué no aprende el alumnado el contenido matemático con la calidad requerida? ¿Qué impide realizar un diagnóstico pormenorizado del aprendizaje de los miembros del grupo estudiantil?

Del desarrollo del acercamiento surge la propuesta de un cuestionario que se convierte en una orientación para la autovaloración crítica y reflexión colectiva del pensamiento profesional pedagógico en el desarrollo de actividades docentes específicas en los contextos formativos. El mismo se estructura con las siguientes preguntas genéricas: 1) ¿Qué es lo que hago para el desarrollo del aprendizaje del contenido en el alumnado? 2) ¿Cómo he llegado a pensarlo así? 3) ¿Qué significa para los miembros del grupo estudiantil la tarea docente que se le asigna para aprender el contenido? 4) ¿Qué podría hacer diferente para alcanzar mejores resultados?

Las personas autoras de este artículo consideraron útil incluir el registro etnográfico obtenido del trabajo de campo realizado y que fue empleado en el desarrollo del grupo de discusión en el cual se realizaron varias sesiones de reflexión y debate hasta llegar a un consenso por los implicados de la fidelidad de los datos obtenidos y de la pertinencia de las buenas prácticas identificadas. El registro etnográfico no persigue una categorización de los criterios y creencias en él recogidos sino socializar unidades de significados en los campos temáticos que son determinantes en la solución del problema y que son expresión de la diversidad de maneras de pensar y actuar de una parte del profesorado. Es también parte de la intención de socializar el registro etnográfico para que otros etnógrafos encuentren en él datos y puedan sacar otras conclusiones no abordadas en este estudio. 
Podrá apreciarse en la lectura del registro etnográfico que en las cuestiones que, en cursiva se plasman en el texto tal y como son usadas por las personas investigadas, deben seguirse realizando análisis críticos hasta superar aspectos que pueden ser mejorados y que en este momento de la investigación no se lograron transformar en las creencias de una parte del profesorado.

Es necesario reiterar que los elementos registrados no son los que predominan en la mayoría de las personas parte de la muestra, sino que como estudio cualitativo de tipo etnográfico se registra la diversidad de formas de pensar sin importa representatividad estadística pues cada docente cuenta para lograr un cambio en la forma de gestionar aprendizaje.

\subsection{Análisis del registro etnográfico}

En las creencias, mitos y preconceptos registrados en el trabajo de campo que conforman el pensamiento pedagógico del profesorado de matemática de Santiago de Cuba para el diseño de su labor pedagógica, existe un mito sobre los manuales conformados para el alumnado, también conocidos como libros de textos en el modelo percibido: el que resuelva todos los ejercicios que están en el libro de texto aprueba cualquier examen. Realmente esto no ha sido comprobado y probablemente no sea posible pues en la asignatura se utilizan cuatro textos uno de ellos básico y tres complementarios que en su totalidad reúnen una gran cantidad de ejercicios.

En la preparación para el ingreso a la Educación Superior en criterio de especialistas es necesario realizar una selección de toda la diversidad de ejercicios que además poseen diferentes niveles de integración de los contenidos. El dilema está en cómo organizar de manera didáctica el trabajo con estos textos para lograr gradación de lo simple a lo complejo abarcando toda la variedad del contenido y vías de solución.

Al referirse al manual y libros complementarios que sirven de bibliografía el profesorado opina que los libros lo tienen todo, solo hay que trabajar con ellos de manera sistemática.

En el trabajo con ejercicios durante la clase opinan los miembros del claustro que se estimula demasiado al que primero hace el ejercicio. Este planteamiento orienta a considerar la revisión, en el modelo percibido, de la creencia organizativa de que los turnos de clases deben tener una duración de cuarenta y cinco minutos. Ello es razón por la cual la prisa en la solución de los ejercicios seleccionados para la clase induzca al docente a premiar la rapidez 
y no ponderar la reflexión que favorezca a los diversos ritmos de aprendizajes presentes en cualquier grupo estudiantil.

Se trasmite por docentes y entre ellos, a los familiares y al alumnado la creencia de que la matemática no es fácil. Con esta idea en el modelo percibido el profesorado ha construido una especie de dispositivo con la cual connota socialmente la asignatura y regula la disciplina del alumnado. El miedo a la asignatura ha sido una de las características percibidas en la realidad escolar que incide en el rendimiento del alumnado y en alguna medida en el diseño de las tareas de aprendizaje por los miembros del claustro en el proceso de gestión de aprendizaje en el que la gradación de los simple a lo complejo y la creación de condiciones previas para la sistematización y adquisición de nuevos conocimientos es esencial. Al preguntar el criterio al profesorado sobre ejercicios, ellos califican de bonitos aquellos en el que el estudiante tiene que realizar mayor cantidad de aplicaciones con vías diferentes para resolverlos convirtiendo en el modelo percibido lo bonito en sinónimo de complejo.

Junto a la práctica de esta creencia se manifiesta como necesidad sentida el reclamo de que hay que motivar para que le guste la matemática. Como una condición en la gestión de aprendizajes a partir de reconocer de que por lo general el alumnado no está motivado. Por tanto, por una parte, se crea una representación escolar de que es difícil la asignatura y por otra, en el discurso se reconoce la necesidad de motivar al alumnado.

La representación de la responsabilidad del aprendizaje es otro de los dilemas que influye en la gestión de aprendizaje. Es común escuchar la expresión docente que el problema de aprender es del alumnado, ellos son los que no saben. Esta creencia en el modelo percibido, al someterla al análisis contiene una verdad relativa pues no es desdeñable el papel que el profesorado desempeña en el logro del aprendizaje de la población estudiantil. Por otra parte, se registra en los grupos de personas que realizan labor directiva otra creencia que se contrapone a la del profesorado: el alumnado no sabe porque el profesorado no le enseña.

En el análisis de los resultados de una comprobación realizada por una instancia de dirección a un grupo de estudiantil el profesorado considera que la causa del resultado es que la forma de preguntar lo perdió en esa prueba. De la dialéctica entre la enseñanza y el aprendizaje, en la relación modelo percibido modelo operatorio, se expresa un dilema cultural que afecta la gestión de aprendizaje de contenidos matemáticos en el contexto santiaguero. 
Existe consenso en el profesorado de que para la preparación del grupo estudiantil en el ingreso a la Educación Superior no se puede perder tiempo en doce grados, hay que comenzar desde septiembre el entrenamiento intensivo.

Otra de las creencias presentes en el modelo percibido es que se aprende haciendo. Si el estudiante tiene problemas en un contenido, hay que dejarles ejercicios suficientes para que ejercite. Ante la complejidad de abstracción que se reconocen en los contenidos de geometría el profesorado con buenos resultados plantea que los ejercicios de geometría hay que explotarlos lo más posible.

Esta idea de aprender con la ejercitación es razón por la cual piensa el profesorado de matemática que en la relación tecnología aprendizaje matemático: hay que prohibir en las clases de matemática el uso de equipos tecnológicos para realizar cálculo, eso no desarrolla el pensamiento. Otros piensan que el uso de equipos en el cálculo no es el problema del aprendizaje, en la calificación, por problemas de cálculo, se pierde uno o dos puntos de veinte que vale la pregunta, eso no es lo que determina la calidad del aprendizaje. Este análisis describe otra de las situaciones que conforma un dilema cultural en el pensamiento profesional pedagógico.

Los miembros del grupo de docentes reconocen en la memoria un aspecto importante en el aprendizaje de la matemática: hay cosas que deben ser aprendidas de memoria, como se aprende una canción. Es indiscutible la validez de esta creencia, solo que la memorización debe ser acompañada de la comprensión y la reflexión crítica cuestión no lograda en la práctica generalizada en la enseñanza.

Los miembros del profesorado de mejores resultados creen que para aprender las propiedades de las figuras geométricas hay que ficharlas, comprenderlas y sistematizarlas a través de ejercicios y conceptos. La forma en que concibe el logro de estos aprendizajes queda expresada en esencia en los elementos subyacentes de expresiones como: los conceptos hay que decírselos primero en las clases. Cuando se lo expliqué me di cuenta que se apropiaron del concepto y su definición. El concepto solo se aprende si se comprende.

Otra de las creencias es que el estudiante que más aprende es el que más participa en clases. En el modelo percibido del profesorado de matemática, se entiende por participar que los participantes se expresen a viva voz durante la clase, su valoración se realiza a través de la manifestación externa y conductual en la realización de tareas docentes. La comprobación de la participación se efectúa generalmente mediante la observación sin poder contactar y profundizar en la valoración de la contribución a partir de la atención y la reflexión que en la 
situación de aprendizaje experimenta el miembro del grupo estudiantil que no se expresa en voz alta.

La participación se conceptualiza en el modelo percibido como protagonismo estudiantil y en él se registra la concepción de que para lograr aprendizaje hay que alcanzar el protagonismo estudiantil en la clase. Los miembros del grupo de docentes de mejores resultados piensan que para que aprenda el alumnado el profesorado no tiene que demostrar que sabe en la pizarra. El o la estudiante debe elaborar ejercicios y resolverlos, seleccionar los ejercicios del libro de texto y justificar porque los selecciona según su situación de aprendizaje.

Al explicar por qué no aprenden más los miembros del grupo estudiantil, en determinados contenidos, se registra la observación por el profesorado de que en ocasiones la persona sabe la propiedad de la figura, pero no le gusta escribir, eso limita sus posibilidades de sistematizar aprendizaje. Cuando se aplica un temario, no siempre el alumnado le da importancia y se esfuerza en realizar cada pregunta con calidad porque no se contempla el resultado en la evaluación.

La modelación de la clase es una de las cuestiones fundamentales en la gestión de aprendizaje matemático. Ante la pregunta qué determina que el estudiante aprenda en la clase, se registran cuatro tendencias en la argumentación dadas en: A) El profesorado da toda la información que se necesita y se la organiza en la pizarra al alumnado para luego sistematizarla. B) El profesorado da toda la información para que el alumnado la organice y aplique a ejercicios. C) El profesorado orienta la búsqueda por el alumnado parcial o total de la información y luego la aplica en ejercicios del libro de texto o creados por los propios miembros del grupo estudiantil. D) El profesorado transita por los diferentes modelos anteriores hasta llegar al $C$.

Dentro de las condiciones necesarias que se reconoce por el profesorado de matemática para el aprendizaje está que para poder enseñar al alumnado tiene que estar en el aula. Para poder lograr calidad en el resultado en el duodécimo grado hay que lograr que se mantengan mañana y tarde en las escuelas en locales con condiciones para su atención por el profesorado.

Otra de las condiciones en las que se expresa consenso es que el profesorado tiene que estar preparado. Para poder alcanzar resultados el profesorado debe dominar el programa de ingreso a la Educación Superior y el de cada nivel educativo para identificar 
dónde comienza el error y poder dar tratamiento. El profesorado debe elaborar ejercicios y resolverlos.

Se creen que la atención a las diferencias individuales es la clave del aprendizaje grupal. Considera el profesorado que la atención a las diferencias individuales es determinante en la preparación para el ingreso a la Educación Superior. Para su concreción se cree que: Si no es bueno el diagnóstico, es mala la atención a las diferencias individuales. Yo al que le pregunto en la clase es al que sé que tiene problemas de aprendizaje así lo atiendo diferenciadamente y actualizo de manera permanente el diagnóstico.

La importancia del diagnóstico y la identificación de la responsabilidad del profesorado de grados anteriores en los diferentes niveles de aprendizajes son argumentos para explicar el rendimiento escolar en el desarrollo del proceso de enseñanza aprendizaje.

Explican miembros del grupo de docentes que: Después que le imparto en clases el contenido organizo la atención a los que no lo aprendieron. Yo incorporo al sistema de clases que voy a impartir los contenidos del sistema anterior que no aprendieron. Cuando un miembro del grupo estudiantil no sabe nada hay que recurrir al plan machaque ${ }^{5}$, con trucos ${ }^{6}$ $y$ de manera individual avanzar poco a poco. Yo concibo un espacio extra al horario para ir barriendo con el programa de ingreso como espacio de sistematización. Yo relaciono el contenido del programa que imparto en la mañana con el del ingreso en la tarde para sistematizar tanto para la prueba de ingreso como para la prueba estatal.

Entre las barreras que identifica el profesorado de matemática para la atención a las diferencias individuales señalan que: como hay que trabajar con todos y traen tantas lagunas se hace más lento el avance. Me siento con dificultades todavía en cómo dar tratamiento a un mismo elemento del conocimiento a personas de diferentes rendimientos académicos porque los de alto resuelven más rápido y marcan el ritmo del tratamiento. Existen otros que discrepan pues: No se puede seguir pensando que el alumnado que viene con lagunas de otros grados, eso limita la posibilidad del profesorado generar soluciones.

Dentro de las estrategias compensatorias existen experiencias en trabajos de tutoría entre pares que es codificada en la expresión: Para atender a los que menos aprenden se crean parejas de equilibrio, casas de estudio.

\footnotetext{
${ }^{5}$ Por plan machaque se entiende en el modelo percibido la repetición del contenido en la explicación docente hasta lograr que se fije el conocimiento que se pretende enseñar. (Nota de los autores)

${ }^{6}$ Entiende el grupo de docentes como trucos los recursos nemotécnicos que crean para facilitar la memorización de definiciones, teoremas, fórmulas reglas, etcétera. (Nota de los autores)

${ }^{7}$ En lenguaje cotidiano del grupo estudiado se entiende por prueba estatal al examen final por el hecho de que no es elaborado por la escuela sino por instancias superiores. (Nota de los autores)
} 
Existe la creencia de que los estudiantes tienen muchas lagunas de conocimientos. En el modelo percibido esta expresión hace referencia a los conocimientos recibidos en cursos anteriores y que debía saber el alumnado, como aseguramiento del nivel de partida de los nuevos contenidos.

De forma simbólica para definir la situación de aprendizaje del alumnado y explicar por qué no aprenden los contenidos plantea el profesorado que: no aprenden porque vienen con muchas lagunas de conocimientos de los grados y niveles anteriores. Si la atención en grados anteriores es mala, en doce ${ }^{8}$ es malo el resultado, porque en un curso no se puede hacer magia. Sin ánimo de justificar el problema: Si el alumnado tiene la base (lo que debía saber) puede aprender el nuevo contenido.

Otro de los argumentos utilizados muestra el nivel de sobreprotección que existe en las creencias del profesorado para hacer ajustes en la gestión de aprendizajes: Ese problema de aprendizaje no ha tenido tratamiento porque el contenido que ya recibió en la secundaria básica todavía no ha sido sistematizado en mi clase. Yo diagnostico solo lo que está dando el alumnado en mi clase o lo que debe saber de lo próximo que voy a dar, si diagnostico todo lo que debe saber el alumnado rápidamente coge miedo a la asignatura. Quiere decir que, en el modelo percibido, lo que el profesorado no ha tratado en el nivel o grado en que trabaja, no tiene por qué saberlo el alumnado, aunque haya sido estudiado en grados anteriores.

La educación en Cuba se estructura en programas de estudios que conforman el currículo general para todo el país, por tanto, es responsabilidad del profesorado cumplir el programa que imparte y que a la par cumplan los objetivos de cada grado y nivel. En esta práctica se cree que: Si paso a otro contenido sin que el alumnado venza este, estoy creando lagunas de conocimiento 9 .

La matemática se aplica a la valoración del desempeño de aprendizaje como un instrumento en el manejo estadístico en el proceso de gestión de aprendizaje por las personas que tienen función directiva y docente. El resultado de aprendizaje es asumido como indicador para valorar el desempeño del profesorado a partir de la creencia de que el alumnado no aprende lo que el profesorado no le enseña. Con este pensamiento existen

\footnotetext{
${ }^{8}$ Doce refiere al grado escolar terminal del nivel preuniversitario. (Nota de los autores)

${ }^{9}$ Lagunas de conocimiento constituye un término de uso cotidiano en la escuela por el profesorado para denotar la ausencia de conocimientos que debieron formarse en etapas anteriores del desarrollo de la persona, los autores no pretenden profundizar de su pertinencia sino reflejar etnográficamente como se representan en el modelo percibido categorizaciones para identificar insuficiencias en el diagnóstico. (Nota de los autores)
} 
cuestionamientos pues plantea el profesorado que: Yo no sé por qué me exigen resultados superiores cada curso si el alumnado cada año son diferentes.

Se observan comportamientos que sobredimensionan el aspecto cuantitativo en la valoración de los resultados de aprendizaje. Por ejemplo, al referir a una prueba diagnóstica aplicada plantearon que: En este examen suspendieron diez, se mantiene el diagnóstico, aunque no son las mismas personas. Para la valoración del resultado de aprendizaje estudiantil no era bueno que suspendieran, pero era peor si la cifra de suspensos era superior a la alcanzada en etapas anteriores.

Se identifica como una relación importante que, la visión que se tiene de cada uno de los miembros del alumnado determina su atención educativa. Otro de los aspectos significativos identificado en el pensamiento del profesorado de matemática es la caracterización que tiene del grupo y de sus integrantes. Por lo general al exponerla prevalecen los siguientes razonamientos: El grupo se caracteriza como promedio porque predominan personas de rendimiento medio y los aventajados ${ }^{10}$ son menos. Caracterizan a los miembros del grupo estudiantil como aprobados, pero con problemas en elementos del conocimiento, listo, suspenso para el ingreso a la Educación Superior. El grupo es bastante estable porque no tiene ausencias y hay un buen comportamiento. El grupo es disciplinado, pero le falta preparación en la asignatura. Para caracterizar al alumnado tengo en cuenta dos variables: rango de notas y elementos del conocimiento. Con estas variables organizo la atención a las diferencias individuales y dar seguimiento al diagnóstico.

En el discurso metodológico se habla de la estrategia para aprender matemática. Sin embargo, no siempre queda claro: ¿La estrategia de quién y para quién? Este aspecto es determinante para poder comprender los niveles de participación e implicación en el diseño y ejecución de la estrategia de trabajo en la gestión de aprendizajes matemáticos. En la medida en que se aplican instrumentos hay que mover la escuela, la estrategia es flexible, no puede ser esquemática. En matemática hay que ir cumpliendo al mismo tiempo los objetivos del programa y los del ingreso.

Enfatizan los docentes que: Para el aprendizaje de la matemática hay palabras de orden: diagnóstico, orientación, seguimiento y control de las actividades de aprendizajes.

10 En el modelo percibido se identifica a estudiantes aventajados a aquellos de alto rendimiento académico (alcanzan niveles de desempeños altos en el aprendizaje de los contenidos). (Nota de los autores) 
Hay que orientar lo que se va controlar. Yo oriento tres tareas en cada clase: una de la clase que di, otra de lo que nivelo ${ }^{11}$ y otra que se antecede al otro contenido.

Otro aspecto que alude el profesorado para explicar la situación de aprendizaje del alumnado es la responsabilidad, ellos creen que a la familia le corresponde la responsabilidad de crear un hábito de estudio en la casa.

\section{Conclusiones}

Con el trabajo de campo realizado no solo se obtiene el registro de las creencias y prácticas con las cuales explica el profesorado de matemática de Santiago de Cuba los resultados en la gestión de aprendizaje sino que se llega a consenso de las estrategias directrices a partir de las buenas prácticas identificadas en el trabajo de campo y argumentadas por el propio profesorado como vía para formar conocimientos duraderos en el alumnado.

Las cualidades resultantes que se obtienen se expresan en determinaciones concretas que fundamentan experiencias valiosas y necesidades de cambio registradas en: conformación de descriptores y registros etnográficos, conformación de criterios de realización, conformación de memorias de soluciones de problemas situacionales y conformación del marco de la nueva gestión. La articulación coherente de las estrategias directrices y las cualidades resultantes se constituyen en contenidos de la gestión pedagógica que dan garantía en la práctica probada de Santiago de Cuba de una manera de formar conocimientos duraderos en los estudiantes.

La investigación realizada constituye un registro del pensamiento pedagógico de los docentes de matemática de cómo desarrollar la gestión de aprendizaje y brinda elementos teóricos y fácticos que permiten el estudio de la dialéctica que se establece entre las creencias y las concepciones de las personas implicadas en el diseño y realización del proceso pedagógico.

En esta propuesta, ya convertida en experiencia sistematizada y fundamentada en la provincia Santiago de Cuba, se transita de la etnografía como método de trabajo de campo a la sistematización de experiencias etnográficas y de esta, se alcanza a incorporar la práctica auto-etnográfica en las herramientas para el perfeccionamiento de la enseñanza de la

\footnotetext{
11 El término es usado en el lenguaje de los docentes para categorizar la acción de compensación de los conocimientos precedentes necesarios para la impartición y asimilación de los nuevos según diseño curricular. (Nota de los autores)
} 
matemática, donde la revisión crítica se convierte en fuente generadora de nuevos conocimientos y prácticas.

El cambio principal y aporte están en el profesorado, en el análisis y observación crítica de su propia actividad, o sea, el conocimiento de la pertinencia de su propia actividad pedagógica profesional, para asumir su cambio en los contextos de formación, cuando estos no le resulten adecuados.

Se convierte la etnografía en un método que se transpone a la pedagogía que pasa por sucesivas transformaciones como saber propio de la antropología, su mutación en saber a enseñar hasta llegar a ser utilizado como saber enseñado útil para generar niveles progresivos de reflexividad en el cual ocurre un proceso de profesionalización de la enseñanza para perfeccionarse por sí mismo como docente, con la ayuda de otros y a través del pensamiento crítico sobre la auto-etnografía. Junto al cambio principal se precisa como el estudio cardinal para lograr la profesionalización: la reflexión sobre la acción pedagógica en tiempo real y la toma de decisión sobre ella de forma genuinamente participativa.

\section{Referencias}

Araya, Sandra. (2002). Las representaciones sociales: Ejes teóricos para su discusión. (Cuaderno de Ciencias Sociales 127). San José, Costa Rica: Facultad Latinoamericana de Ciencias Sociales (FLACSO).

Barquín, Javier. (1991). La evolución del pensamiento pedagógico del profesor. Revista de Educación, (294), 245-274.

Berger, Peter. y Luckmann, Thomas. (1991). La construcción social de la realidad. Buenos Aires, Argentina: Amorrortu.

Callejo, Javier. (2001). El grupo de discusión: introducción a una práctica de investigación. Barcelona: Ariel Practicum.

Comaroff, John. y Comaroff, Jean. (1992). La etnografía y la imaginación histórica. Boulder: West view Press.

Escobar, Arturo. (2014). La invención del desarrollo. Popayán, Colombia: Universidad del Cauca.

Geertz, Clifford. (2003). Descripción densa: hacia una teoría interpretativa de la cultura. En Paul Bohannan y Mark Glazer (eds.), Antropología lecturas (pp. 545-568). España: McGraw-Hill.

Jodelet, Denise. (1984). La representación social: fenómenos, conceptos y teoría. En Serguei Moscovici (ed.), Psicología social II. Pensamiento y vida social. Psicología social y problemas sociales. Barcelona-Buenos Aires-México: Paidós. 
Justis, Osmany, Almestro, Sandra y Silva, Olga. (2017). Pedagogía para el desarrollo socioemocional. A propósito de la gestión de aprendizaje en el contexto santiaguero. Revista Iberoamericana de Educación, 75(2), 109-126

Justis, Osmany. (2013). La cultura escolar en la toma de decisiones del grupo de docentes en el proceso pedagógico del preuniversitario (Tesis inédita, presentada en opción al grado científico de Doctor en Ciencias Pedagógicas). Universidad de Ciencias Pedagógicas Frank País García, Santiago de Cuba.

Justis, Osmany. (2019). Procedimientos para la gestión de aprendizaje en la ayuda metodológica. Una propuesta en el marco del Tercer Perfeccionamiento del Sistema Nacional de Educación en Santiago de Cuba. Editorial Académica Española.

Krueger, Richard. (1991). El grupo de discusión. Guía práctica para la investigación aplicada. Madrid: Pirámide.

Martínez, Jaume. (1988). Pensamiento del profesor y renovación pedagógica. Revista Investigación en la escuela, (4), 13-18.

Moscovici, Serguei. (1979). El psicoanálisis, su imagen y su público. Buenos Aires, Argentina: Huemul S.A.

Moscovici, Serguei. (1981). La era de las Multitudes. Un tratado histórico de psicología de masas. México: Fondo de Cultura Económica.

Moscovici, Serguei. (1984). El campo de la psicología social. En Moscovici Serguei. La psicología social l. Barcelona, España: Paidós.

Moscovici, Serguei. (1991). La Sicología Social I. Barcelona, España: Paidós.

Serrano, Rocío. (2010). Pensamientos del profesor: un acercamiento a las creencias y concepciones sobre el proceso de enseñanza-aprendizaje en la Educación Superior. Revista de Educación, (352), 267-287.

Soto, Rosa Cristina, Juárez, Fernando y García, José Martín (2011). ¿Creencia o conocimiento pedagógico? Revista Universitaria, Hecho en casa, (08). Recuperado de, http://educa.upnvirtual.edu.mx/educapdf/rev8/soto-008.pdf

Wolf, Eric. (1982). Europa y la gente sin historia. México D.F.: Fondo de Cultura Económica,

Zamora, Paula y Balmaceda, Christian (2015). Creencias epistemológicas en profesores que postulan al Programa de Acreditación de Excelencia Pedagógica: Análisis descriptivos y comparativos entre profesores que se desempeñan en los diferentes niveles de enseñanza. Revista Estudios Pedagógicos, 41(2), 107-125. 
Revista indizada en

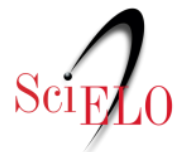

redalyc satindex

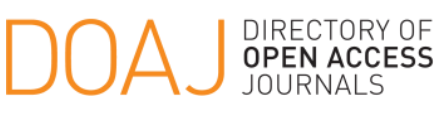

Distribuida en las bases de datos:

- Dialnet

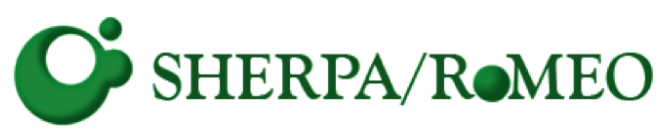

REDIB

Red Iberoamericana

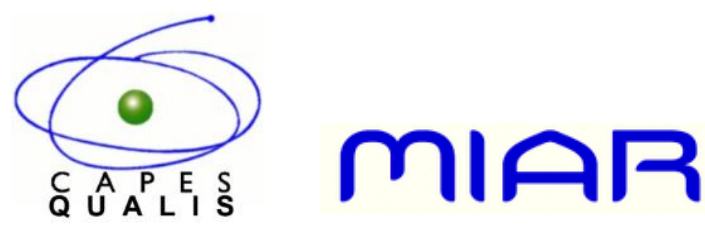

